\title{
Study of baryon pair production at BESIII
}

\section{Xiongfei Wang ${ }^{a, b, *}$}

${ }^{a}$ School of Physical Science and Technology, Lanzhou University, No. 222 South Tianshui Road, Lanzhou 730000, People's Republic of China

${ }^{b}$ Lanzhou Center for Theoretical Physics, Frontiers Science Center for Rare Isotopes and Key Laboratory of Theoretical Physics of Gansu Province, Lanzhou University, No. 222 South Tianshui Road, Lanzhou 730000, People's Republic of China

E-mail: wangxiongfei@lzu.edu.cn

The BESIII detector at BEPCII Collider has collected the world's largest data sets. Some of recent results on the baryon pair production in charmonium(-like) decays, $J / \psi, \psi(3686)$, etc., and in the positron-electron annihilation are reported, which covers the observation of more charmonium modes, the measurement of triply-strange baryon polarization, most accurate measurements of nucleon form factor, and more precise studies for baryon pair production near threshold. These results provide a rich laboratory to test the standard model, such as probing the limitation of the quark models and spotting unrevealed aspects of the QCD description of the structure of hadron resonances.

\footnotetext{
*** 10th International Workshop on Charm Physics (CHARM2020), ***

***31 May - 4 June, 2021 ***

*** Mexico City, Mexico - Online ***
}

\footnotetext{
${ }^{*}$ Speaker
} 


\section{Introduction}

The studies of the baryon pair production in charmonium(-like) decays and in $e^{+} e^{-}$annihilation provide a rich laboratory to test the standard model, such as probing the limitation of the quark models and spotting unrevealed aspects of the QCD description of hadron resonances [1-7]. Figure 1 shows the Feynman diagram for the baryon pair production in charmonium(-like) decay and in $e^{+} e^{-}$ annihilation.
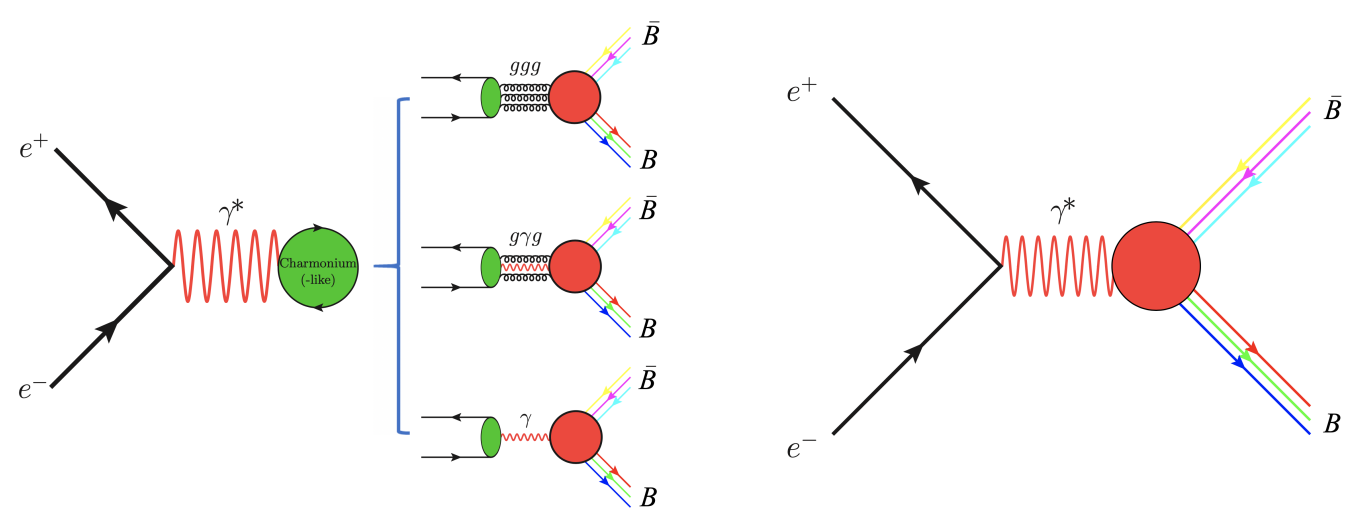

Figure 1: The baryon $(B)$ pair production in $e^{+} e^{-}$annihilation via charmonium(-like) decay for three gluons process, the virtual photon process and mixed two gluons and one virtual photon (left). The baryon $(B)$ pair production in $e^{+} e^{-}$annihilation via one photon exchange (right).

Shortly after the discovery of $J / \psi$, Appelquist and Politer [8] proposed that if there existed a heavy charm quark, it should form a nonrelativistic $c \bar{c}$ bound state with a similar spectrum of energy levels to the positronium. It then was called charmonium by taking into account beautifying the language. Subsequently, more and more charmonium(-like) states $\eta_{c}(1 S), \chi_{c J}, \psi(2 S)$, $\psi(3773), \psi_{2}(3823), \psi_{3}(3842), X(3872), \psi(4040), Y(4260), Y(4360), \psi(4415), Y(4660)$, etc., were found [9]. Here the ones $X(3872), Y(4260), Y(4360) Y(4660)$, etc., were originally thought to be charmonium states, but some of current evidences and theoretical predictions suggested more exotic explanations, such as a tetraquark state, a molecule or a hybird meson [10,11], which sometimes are so-called charmonium-like states. Beside them, other states $\eta_{c}(1 S), \chi_{c J}, J / \psi, \psi(2 S)$, $\psi(3773), \psi_{2}(3823), \psi_{3}(3842), \psi(4040), \psi(4160), \psi(4415)$, etc., the experimental informations are basically consistent with the predictions from the potential models and lattice QCD [12-17], which were thought to be pure charmonium states, it is so-called conventional charmonium states.

Baryon is any of a family of subatomic particles composed of three quarks or three antiquarks. They are generally more massive than mesons, and interact with each other via the strong force. The established baryon states are described by 3-quark configuration with the zero total color charge. According to their spin difference, they can be divided into two types as shown in Figure 2, one is the $1 / 2$ baryon states, the other is the $3 / 2$ baryon states. In BESIII experiment, we mainly focus on the studies of baryon octet and decuplet, and even extend to study charmed baryons, $\Lambda_{c}^{+}$, $\Sigma_{c}$, etc.. 

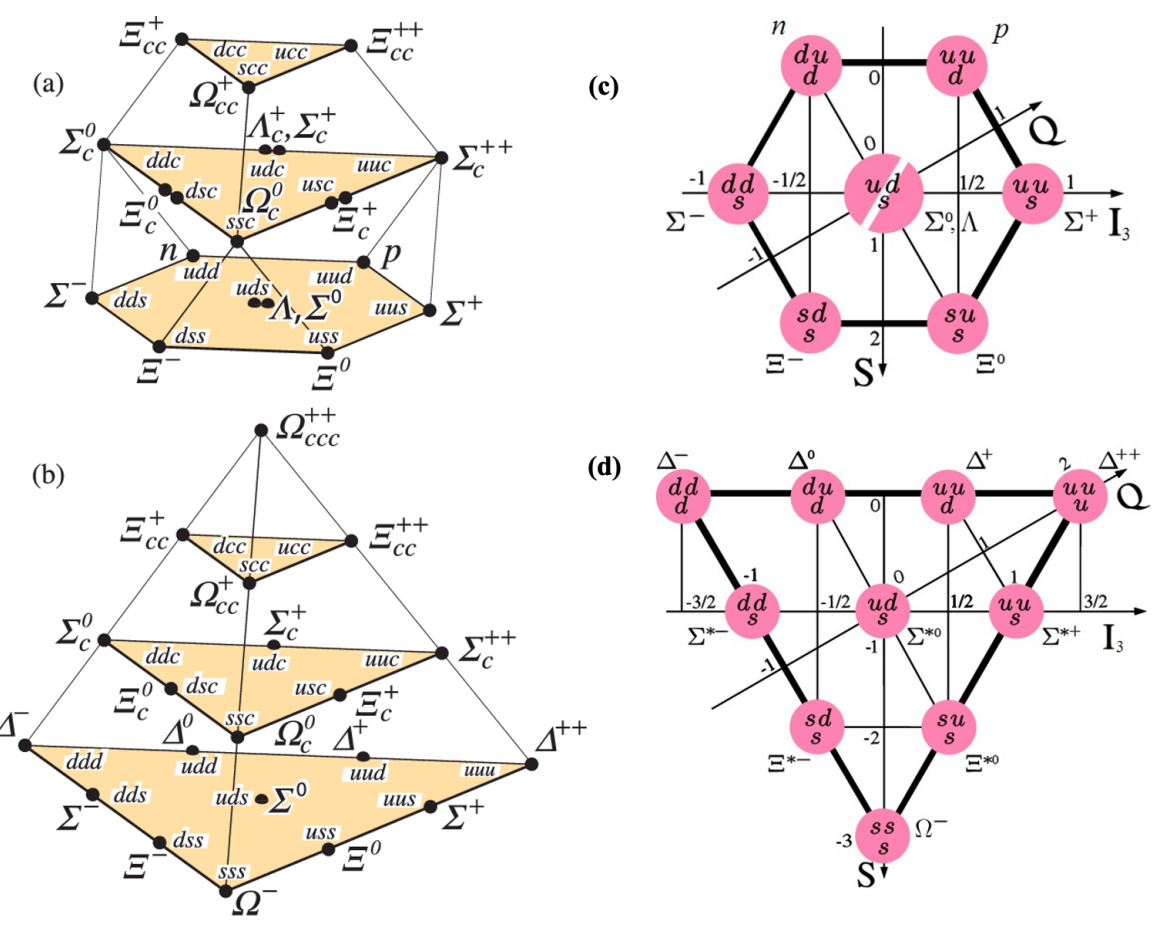

Figure 2: Baryon family with $1 / 2$ spin states (a) and $3 / 2$ spin states (b). Baryon octet with a spin of $1 / 2$ (c) and baryon decuplet with a spin of $3 / 2$.

\section{The BESIII experiment}

The BESIII experiment is dedicated to the $\tau$-charm physics at the Beijing Electron Position Collider (BEPCII) [18]. The BESIII detector [19] shown in Figure 3 records symmetric $e^{+} e^{-}$ collisions provided by the BEPCII storage ring [18], which operates with a peak luminosity of $1 \times 10^{33} \mathrm{~cm}^{-2} \mathrm{~s}^{-1}$ in the center-of-mass $(\mathrm{CM})$ energy range from 2.0 to $4.7 \mathrm{GeV}$. Up to now, the

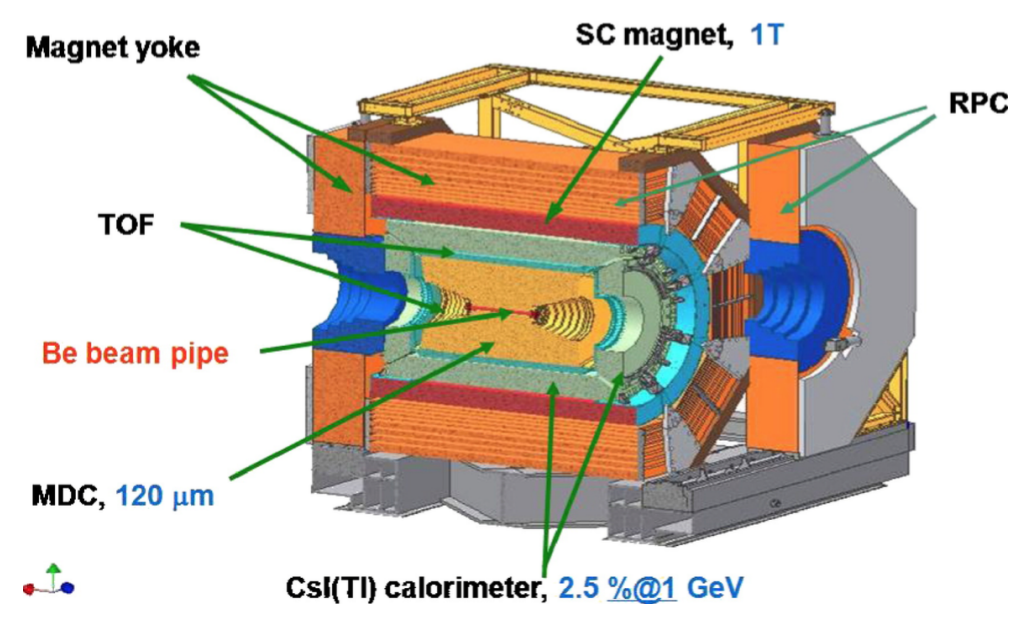

Figure 3: The BESIII detector with the main components [19].

BESIII detector due to the excellent performance has collected the world largest data samples [20], 
i.e. $1.0 \times 10^{10} \mathrm{~J} / \psi$ events [21, 22], $448 \times 10^{-6} \psi(2 S)$ events [23], $2.9 \mathrm{fb}^{-1} \psi(3773)$ events [24], $1.0 \mathrm{fb}^{-1} \mathrm{R}$ scan events [25] and $5.0 \mathrm{fb}^{-1} \mathrm{XYZ}$ data events [26].

\section{Recent results}

\subsection{Baryon pair production in charmonium decay}

3.1.1 $J / \psi \rightarrow \Xi(1530)^{-} \bar{\Xi}^{+}+c . c$.

The $\Xi$ and $\Xi(1530)$ hyperons are regarded as $S U(3)$ octet with a spin of $1 / 2$ and decuplet with a spin of $3 / 2$ as shown in Figure 4 . In the context of the SU(3)-flavor symmetry [2730], the process $J / \psi \rightarrow \Xi(1530) \bar{\Xi}$ should be highly suppressed. However, many experimental results indicate the presence of flavor SU(3) symmetry breaking [9]. Besides, the electromagnetic transition of decuplet to octet hyperons, such as $\Xi(1530)^{-} \rightarrow \gamma \Xi^{-}$, is a very sensitive for probing their internal structures [30-33]. According to a relativistic quark model, the partial width for the decay $\Xi(1530)^{-} \rightarrow \gamma \Xi^{-}$is predicted to be $3.1 \mathrm{keV}$ [30]. Recently, the BESIII experiment presented a more accurate measurement for the branching fraction of $J / \psi \rightarrow \Xi(1530)^{-} \bar{\Xi}^{+}+$c.c. and a search for the the electromagnetic transition process $\Xi(1530)^{-} \rightarrow \gamma \Xi^{-}$, based on 1310 million $J / \psi$ events [34]. Figure 4 shows the distributions of $M_{\pi^{-} \Lambda}^{\text {recoil }}, M_{\gamma \Xi^{-}}$, and $\cos \theta$. The branching fractions for $J / \psi \rightarrow \Xi(1530)^{-} \bar{\Xi}^{+}+$c.c. and $\Xi(1530)^{-} \rightarrow \gamma \Xi^{-}$are measured to be $\mathcal{B}(J / \psi \rightarrow$ $\Xi(1530)^{-} \bar{\Xi}^{+}+$c.c. $)=(3.17 \pm 0.02 \pm 0.08) \times 10^{-4}$ and $\mathcal{B}\left(\Xi(1530)^{-} \rightarrow \gamma \Xi^{-}\right)<3.7 \%$. Angular distribution parameter for $J / \psi \rightarrow \Xi(1530)^{-} \bar{\Xi}^{+}+c . c$. is determined to be $\alpha=0.21 \pm 0.04 \pm 0.06$. Theses results are consistent with the previous measurements and the theoretical prediction [9], provide more experimental information for understanding the theoretical models, such as meson cloud effects [30] and octet-decuplet mixing mechanism [31].
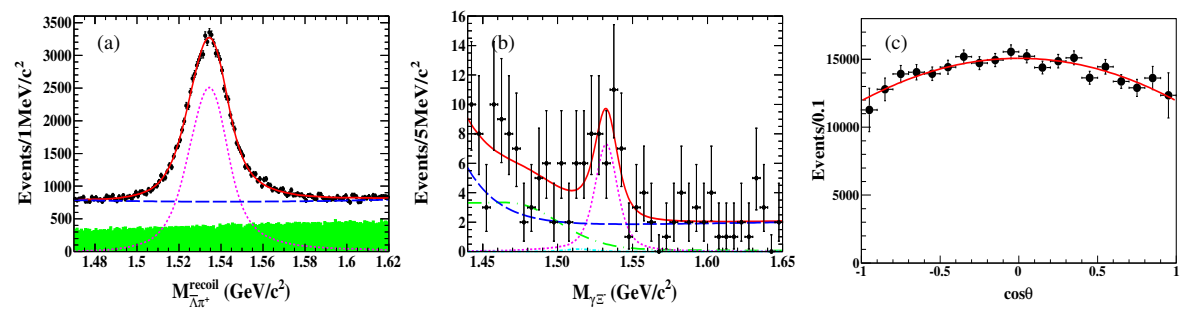

Figure 4: Fit to $M_{\pi^{-} \Lambda}^{\text {recil }}$ (a) and $M_{\gamma \Xi^{-}}$(b), and $\cos \theta$ (c) [34]. The red solid line is the fit result, the pink dotted line denotes the signal, the blue long-dashed one represents the fitted background, and the green-shaded histogram represents the normalized $\bar{\Xi}^{+}$mass sideband events.

\subsection{2 $\psi(3686) \rightarrow \Xi(1530)^{-} \bar{\Xi}(1530)^{+}$and $\Xi(1530)^{-} \bar{\Xi}^{+}+$c.c.}

The baryon pair production in charmonium decay, such as $\psi(3686)$, can be used to test the perturbative QCD, especially by studying the angular distribution for the baryon pair production in charmonium decay, it can also confront the various theoretical models, such as quark mass effects [35] and electromagnetic effects [36]. Recently, the BESIII experiment presented the observation of $\psi(3686) \rightarrow \Xi(1530)^{-} \bar{\Xi}(1530)^{+}$and $\psi(3686) \rightarrow \Xi(1530)^{-} \bar{\Xi}^{+}+$c.c. based on 448 million 
$J / \psi$ events with the statistical significances of more than $10 \sigma$ and $5 \sigma$ using a single baryon tag strategy [37]. Figure 5 shows the recoiling mass spectra of $\pi^{-} \Xi^{0}$ and $\pi^{0} \Xi^{-}$and the distributions of $\cos \theta_{B}$. The branching fractions for both processes are measured to be $\mathcal{B}\left(\psi(3686) \rightarrow \Xi(1530)^{-} \bar{\Xi}(1530)^{+}\right)$ $=(11.45 \pm 0.40 \pm 0.59) \times 10^{-5}$ and $\mathcal{B}\left(\psi(3686) \rightarrow \Xi(1530)^{-} \bar{\Xi}^{+}\right)=(0.70 \pm 0.11 \pm 0.04) \times 10^{-5}$. The observation of $\psi(3686) \rightarrow \Xi(1530)^{-} \bar{\Xi}^{+}+$c.c. further confirms the generality of SU(3) flavor symmetry breaking. Besides, the angular distribution parameter for $\psi(3686) \rightarrow \Xi(1530)^{-} \bar{\Xi}(1530)^{+}$is measured to be $\alpha=0.40 \pm 0.24 \pm 0.06$, which agrees roughly with the theoretical prediction [35, 36] within the uncertainty of $1 \sigma$. It means that the modes for quark mass and electromagnetic effects based on the first order calculation may explain the decay property of charmonium into the hyperons.
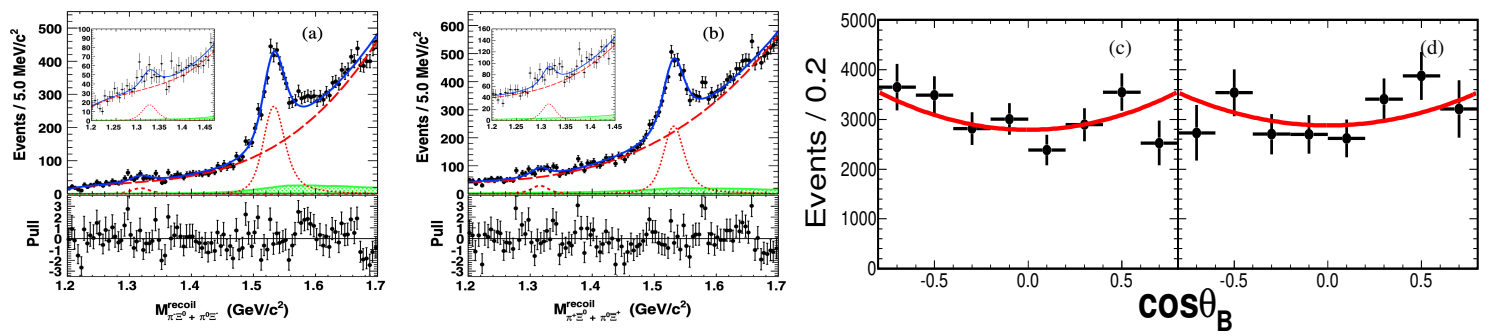

Figure 5: Fit to $M_{\pi \Xi}^{\text {recoil }}$ and $\cos \theta_{B}$ for the $\Xi(1530)^{-}$reconstruction (a,c) and the $\bar{\Xi}(1530)^{+}$reconstruction $(b, d)$. Dots with error bars are data, the blue solid lines show the fit result, the red short-dashed lines are for signal, the red long-dashed ones are for the smooth background, and the green hatched ones are for wrong combination background.

\subsection{3 $\psi(3686) \rightarrow \Omega^{-} \bar{\Omega}^{+}$}

A key feature of the quark model and the eightfold way is that the $\Omega^{-}$baryon has a spin of $3 / 2$ [38, 39], which has never been unambiguously confirmed by experiments. Based on 448 million $\psi(3686)$ events, the BESIII experiment performs the precise study of the angular distribution for $\Omega^{-}$baryon pair production in $\psi(3686)$ decay, and found that the hypothesis of $\Omega^{-}$has a spin of $3 / 2$ with a statistical significance larger than $14 \sigma$ over a spin of $1 / 2$. The spin of the $\Omega^{-}$to be $3 / 2$ is firstly established and independent of the model-based assumptions. The helicity amplitude of $\psi(3686) \rightarrow \Omega^{-} \bar{\Omega}^{+}$and the decay parameter of $\Omega^{-} \rightarrow K^{-} \Lambda, \phi_{\Omega^{-}}$, are measured for the first time. With the measured helicity amplitudes, the $\cos \theta_{\Omega^{-}}$dependence of the multipolar polarization operators as shown in Figure 6 is calculated, where for the process of $e^{+} e^{-} \rightarrow \psi(3686) \rightarrow \Omega^{-} \bar{\Omega}^{+}$, the $\Omega^{-}$baryon not only has vector polarization $\left(r_{1}\right)$, but also has quadrupole $\left(r_{6}, r_{7}, r_{8}\right)$ and even includes octupole $\left(r_{10}, r_{11}\right)$ polarization contributions [40, 41]. Besides, the branching fraction and angular distribution for $\psi(3686) \rightarrow \Omega^{-} \bar{\Omega}^{+}$are measured to be $\mathcal{B}\left(\psi(3686) \rightarrow \Omega^{-} \bar{\Omega}^{+}\right)=(5.85 \pm 0.12 \pm 0.25) \times 10^{-5}$ and $\alpha_{\psi(3686)}=0.24 \pm 0.10$ and consistent with the previous measurements [42, 43], and with high precision [9].

\subsection{Baryon pair production in $e^{+} e^{-}$annihilation}

\subsection{1 $e^{+} e^{-} \rightarrow n \bar{n}$ and $p \bar{p}$}

The neutron $(n, p)$ is a bound states of three valence quarks and a neutral sea consisting of gluons and quark-antiquark pairs. The study of nucleon structure plays a important role for understanding 

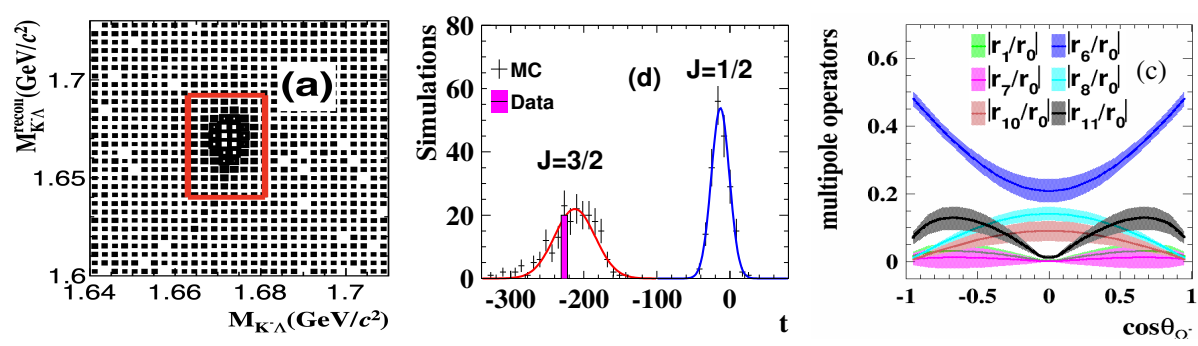

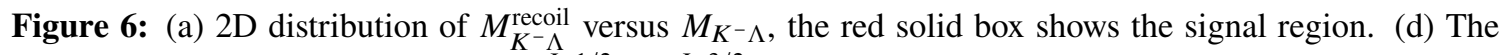
distribution of the test statistic $t=S^{J=1 / 2}-S^{J=3 / 2}$ with MC simulations performed under the $J=1 / 2$ (right peak) and $J=3 / 2$ (left peak) hypotheses. (c) The $\cos \theta_{\Omega^{-}}$dependence of the multipolar polarization operators. The solid lines represent the central values, and the shaded areas are one standard deviation.

strong interactions. In other words, the electromagnetic structure of hadrons, parametrized in terms of electromagnetic form factors (EMFFs), provides a key to understand QCD effects related to the calculation. Recently, the BESIII experiment presented the measurements of Born cross sections and EMFFs for the process of $e^{+} e^{-} \rightarrow n \bar{n}$ using $648 \mathrm{pb}^{-1}$ data events collected at the eighteen energy points between 2.0 and $3.08 \mathrm{GeV}$ [44], and for the process $e^{+} e^{-} \rightarrow p \bar{p}$ using 7.5 $\mathrm{fb}^{-1}$ data events collected at seven energy points from 3.773 to $4.600 \mathrm{GeV}$ based on a initial state radiation (ISR) technique [45]. Figure 7 and 8 show the results for $e^{+} e^{-} \rightarrow n \bar{n}$ and $p \bar{p}$ between these works and previous measurements and theoretical prediction. The results for Born cross section and effective form factor (EFF) are in a good agreement with the measurements from the previous experiments [9], in contradiction to the FENICE and PS170 results. Obvious oscillating structures for both processes are found, which could be attributed to an interference effect involving rescattering processes in the final state [46] or to be independent of resonant structures [47], which could be clarified when the large data sample is available in the future.
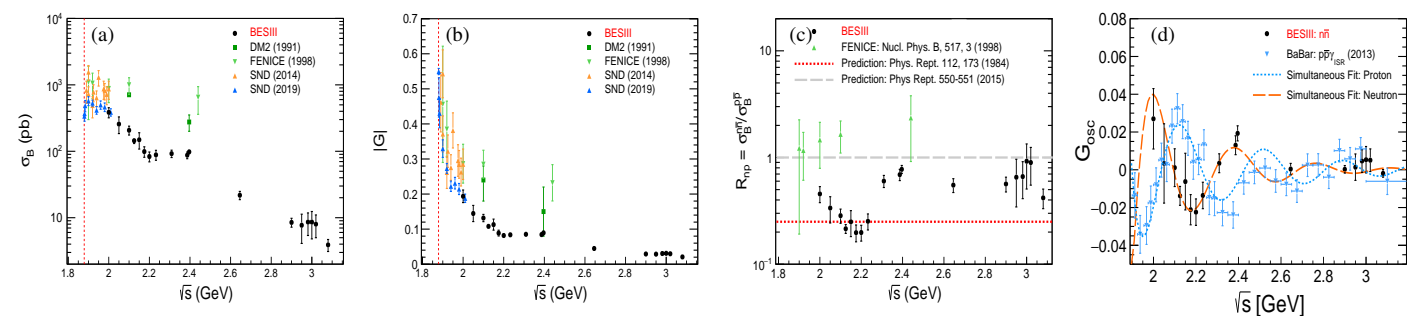

Figure 7: The results of $e^{+} e^{-} \rightarrow n \bar{n}$ for Born cross section (a), effective form factor (b), Born cross section ratio (c) and fit to the deviation of the nucleon EFF from the dipole law (d), between this work and previous results and theoretical prediction.

\subsection{2 $e^{+} e^{-} \rightarrow \Sigma^{ \pm} \bar{\Sigma}^{\mp}$}

The hyperons with one or more strange quarks can provide additional dimensions to study nucleon structures [48-50], and also offer valuable insight into the behavior of the lighter up and down quarks in different environments. Using the data collected by BESIII experiment at eight $\mathrm{CM}$ energies between 2.3864 and $3.02 \mathrm{GeV}$, the Born cross sections and EMFFs of $e^{+} e^{-} \rightarrow \Sigma^{ \pm} \bar{\Sigma}^{\mp}$ are measured for the first time in the off-resonance region. Born cross sections near threshold are 

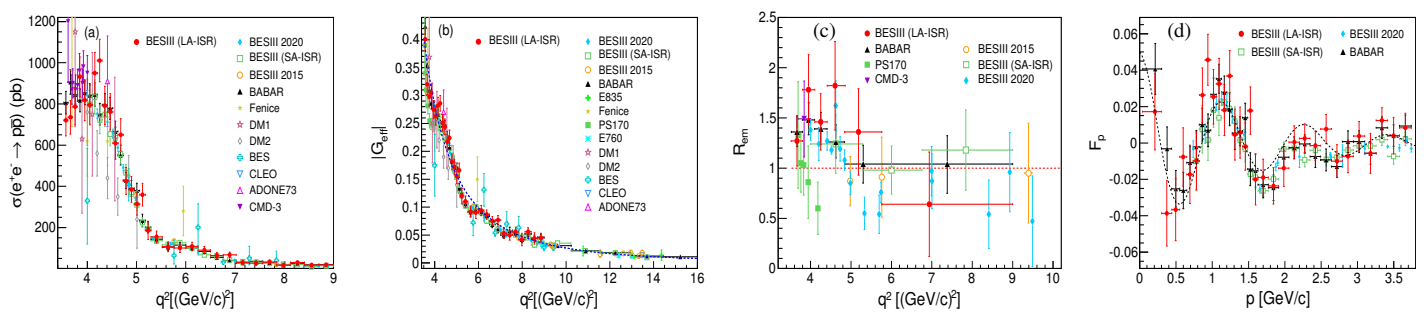

Figure 8: The results of $e^{+} e^{-} \rightarrow p \bar{p}$ between this work and previous results and prediction for Born cross section (a), EFF (b), $\left|G_{E} / G_{M}\right|$ (c) and the proton EFF as a function of the relative momentum (d).

measured for $e^{+} e^{-} \rightarrow \Sigma^{+} \bar{\Sigma}^{-}$and $e^{+} e^{-} \rightarrow \Sigma^{-} \bar{\Sigma}^{+}$to be $58.2 \pm 5.9_{-2.6}^{+2.8} \mathrm{pb}$ and $2.3 \pm 0.5 \pm 0.3 \mathrm{pb}$, respectively, which disagrees with the point-like expectations near threshold, $848\left(m_{p} / m_{B}\right)^{2}$ pb for the proton [51, 52]. The cross section line-shapes for both processes are fitted by pQCD-dirven functions as shown in Figure 9. The Born cross section ratio for both modes is determined to be $9.7 \pm 1.3$, which disagrees with each other within the sector of isospin conservation and the theoretical models [53, 54]. The EMFFs ratio $R_{e m}$ of the $\Sigma^{ \pm}$is also determined at $\sqrt{s}=2.396 \mathrm{GeV}$ based on a study of angular distribution as shown in Figure 9. These informations provide precision experimental input for understanding the $\Sigma^{ \pm}$structure and $\Sigma^{ \pm} \bar{\Sigma}^{\mp}$ pair production near threshold.
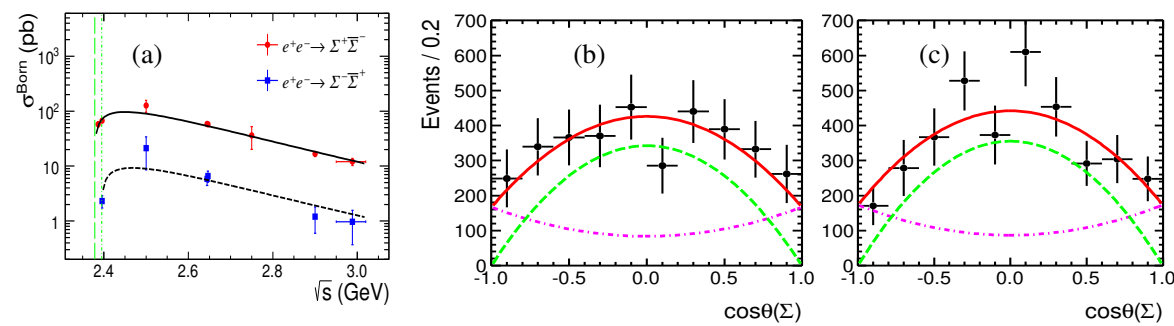

Figure 9: Fit to Born cross sections (a) and angular distributions (b, c) for $e^{+} e^{-} \rightarrow \Sigma^{ \pm} \bar{\Sigma}^{\mp}$.

\subsection{3 $e^{+} e^{-} \rightarrow \Xi^{-} \bar{\Xi}^{+}$and $\Xi^{0} \bar{\Xi}^{0}$ near threshold}

In the past few decades, many experiments have observed non-vanishing cross sections or enhancements for the near-threshold region in the production cross section of nucleon pairs in $e^{+} e^{-}$collisions, which have attracted much interest and driven many theoretical studies. In the context of QCD and even our understanding of the quark-gluon structure of hadrons, it is particularly interesting to probe these anomalous phenomena in the baryon sector [55-58]. Recently, the BESIII experiment presented high precision studies for possible threshold enhancement in the processes $e^{+} e^{-} \rightarrow \Xi^{-} \bar{\Xi}^{+}$and $\Xi^{0} \bar{\Xi}^{0}$, which is based on a single baryon tag method using the $500.0 \mathrm{pb}^{-1}$ data events collected at $\mathrm{CM}$ energies between 2.644 and $3.08 \mathrm{GeV}[59,60]$. The measured Born cross sections are fitted with a pQCD-driven energy power function, which tend to zero near threshold and no obvious threshold enhancement is observed. The ratio of the measured Born cross sections for both modes is within $1 \sigma$ of the expectation of isospin symmetry with the current level of experimental precision. These results provide the insight into the nature of hyperon pair production in $e^{+} e^{-}$annihilation near threshold. Figure 10 shows the results of $e^{+} e^{-} \rightarrow \Xi^{-} \bar{\Xi}^{+}$and $\Xi^{0} \bar{\Xi}^{0}$ for the fitted Born cross sections with the different assumptions and the ratio. 

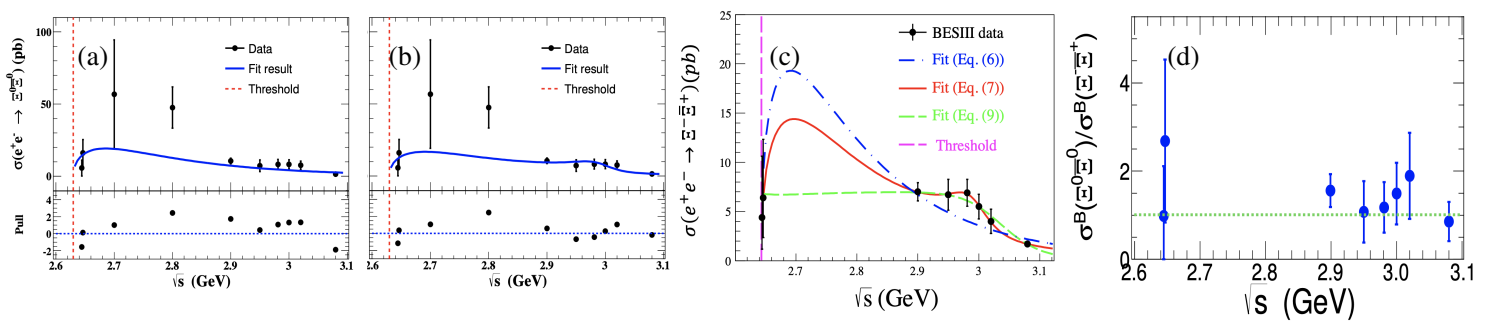

Figure 10: Fit to the Born cross sections for $e^{+} e^{-} \rightarrow \Xi^{0} \bar{\Xi}^{0}(\mathrm{a}, \mathrm{b})$ and $\Xi^{-} \bar{\Xi}^{+}$(c) at the CM energy between 2.644 and $3.080 \mathrm{GeV}$. The ratio of Born cross sections between both modes bottom for eight energy points from 2.6444 to $3.0800 \mathrm{GeV}$ (d).

\subsection{4 $e^{+} e^{-} \rightarrow \Xi^{-} \bar{\Xi}^{+}$above open charm threshold}

The study of the baryon pair production above the open charm threshold in $e^{+} e^{-}$annihilations provides important information for understanding the nature of charmonium-like states [61]. The energy scan above the open charm threshold will be a good search-ground for studying the nature of these charmonium-like states. Recently, the BESIII experiment performed a study of the process $e^{+} e^{-} \rightarrow \Xi^{-} \bar{\Xi}^{+}$based on a single baryon tag technique using a total of $11.0 \mathrm{fb}^{-1}$ of $e^{+} e^{-}$collision data in the CM energy between 4.009 and $4.6 \mathrm{GeV}$ [62]. The Born cross section and the EFFs in this range are measured for the first time. To understand the enhancement near $4.26 \mathrm{GeV}$ well, a fit to the dressed cross section for the $e^{+} e^{-} \rightarrow \Xi^{-} \bar{\Xi}^{+}$process with the assumptions of a power function plus a $Y(4230)$ or $Y(4260)$ resonance is performed, no obvious significances for the processes $Y(4230)$ or $Y(4260) \rightarrow \Xi^{-} \bar{\Xi}^{+}$are observed, the upper limits on the products of the electronic partial width and the branching fractions of $Y(4230)$ and $Y(4260) \rightarrow \Xi^{-} \bar{\Xi}^{+}$are measured to be $\Gamma_{e e} \mathcal{B}_{Y(4230)}<0.33 \times 10^{-3} \mathrm{eV}$ and $\Gamma_{e e} \mathcal{B}_{Y(4260)}<0.27 \times 10^{-3} \mathrm{eV}$ at $90 \% \mathrm{CL}$, which provides more experimental information to understand the nature of $Y(4260)$ [63-67]. Besides, an excited $\Xi$ baryon at $1820 \mathrm{MeV} c^{2}$ is observed with a statistical significance of $5.1 \sigma$ including the systematical uncertainty, the mass and width are measured to be $M=(1825.5 \pm 4.7 \pm 4.7) \mathrm{MeV} / c^{2}$ and $\Gamma=$ $(17.0 \pm 15.0 \pm 7.9) \mathrm{MeV}$, which are consistent with the mass and width of $\Xi(1820)^{-}$obtained from PDG [9] within $1 \sigma$ uncertainty. Figure 11 shows the fitted dressed cross section of $e^{+} e^{-} \rightarrow \Xi^{-} \bar{\Xi}^{+}$ and the recoil mass spectrum of $\pi \Lambda$.
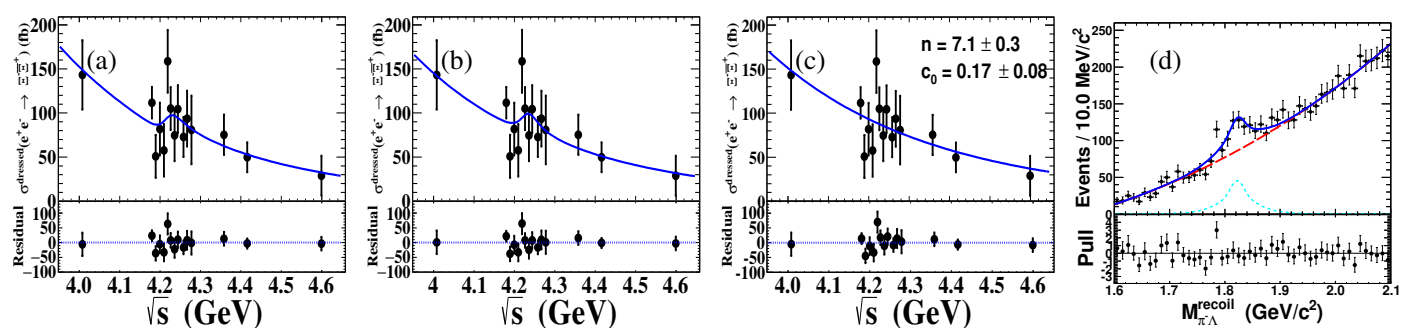

Figure 11: Fit to the dressed cross section with the assumptions of a power function plus a $Y(4230 / 4260)$ resonance function $(\mathrm{a} / \mathrm{b})$, and without resonance $(\mathrm{c})$. Fit to the recoil mass spectra of $\pi \Lambda$ for sum of all energy points (d). 


\section{Summary}

The BESIII experiment is successfully operating since 2008 and has collected the largest data samples in the $\tau$-charm physics region. A lots of results on the baryon pair production in charmonium(-like) decays, such as $J / \psi, \psi(3686)$ decays and in the positron-electron annihilation were achieved. These experimental informations provide a rich laboratory to test the standard model, such as probing the limitation of the quark models and spotting unrevealed aspects of the QCD description of the hadron structure. It is expected that more results for baryon pair production in charmonium(-like) decay and in $e^{+} e^{-}$annihilation will be made at BESIII experiment in the coming future.

\section{Acknowledgements}

This work is supported in part by National Key Research and Development Program of China under Contracts Nos. 2020YFA0406400, 2020YFA0406403; National Natural Science Foundation of China (NSFC) under Contracts Nos. 11905236, 12075107, 12047501; Fundamental Research Funds for the Central Universities under Grants No. lzujbky-2021-sp24.

\section{References}

[1] S. J. Brodsky and G. P. Lepage, Phys. Rev. D 24, 2848 (1981).

[2] N. G. Stefanis and M. Bergmann, Phys. Lett. B 304, 24 (1993).

[3] J. Bolz and P. Kroll, Eur. Phys. J. C 2, 545 (1998).

[4] M. Ablikim et al. (BESIII Collaboration), Phys. Rev. D 87, 032007 (2013).

[5] M. Ablikim et al. (BESIII Collaboration), Phys. Lett. B 770, 217-225 (2017).

[6] M. Ablikim et al. (BESIII Collaboration), Phys. Rev. D 93, 072003 (2016).

[7] X. F. Wang, B. Li, Y. N. Gao and X. C. Lou, Nucl. Phys. B 941, 861-867 (2019).

[8] T. Appelquist et al., Phys. Rev. Lett. 34, 43 (1975); Phys. Rev. D 12, 1404 (1975).

[9] P. A. Zyla et al. (Particle Data Group), PTEP 2020, 083 C01 (2020).

[10] C. Z. Yuan, Int. J. Mod. Phys. A 33, 1830018 (2018).

[11] F. K. Guo et al., Rev. Mod. Phys. 90, 015004 (2018).

[12] S. Godfrey and N. Isgur, Phys. Rev. D 32, 189 (1985).

[13] T. Barnes et al., Phys. Rev. D 72, 054026 (2005).

[14] T. Burch et al., Phys. Rev. D 81, 034508 (2010).

[15] L. Liu et al. (Hadron Spectrum Collaboration), JHEP 1207, 126 (2012). 
[16] N. Brambilla et al., Eur. Phys. J. C 71, 1534 (2011).

[17] X. F. Wang, EPJ Web Conf. 202, 02002 (2019).

[18] C. H. Yu et al., Proceedings of IPAC2016, Busan, Korea, 2016.

[19] M. Ablikim et al. (BESIII Collaboration), Nucl. Instrum. Meth. A 614, 345 (2010).

[20] M. Ablikim et al. (BESIII Collaboration), Chin. Phys. C 44, 040001 (2020).

[21] With the same method (see Ref. [22] for more details), the preliminary number of $J / \psi$ events is determined to $1.0 \times 10^{10}$ with an uncertainty of $0.4 \%$.

[22] M. Ablikim et al. (BESIII Collaboration), Chin. Phys. C 41, 013001 (2017).

[23] M. Ablikim et al. (BESIII Collaboration), Chin. Phys. C 42, 023001 (2018).

[24] M. Ablikim et al. (BESIII Collaboration), Chin. Phys. C 37, 123001 (2013).

[25] M. Ablikim et al. (BESIII Collaboration), Chin. Phys. C 41, 063001 (2017).

[26] M. Ablikim et al. (BESIII Collaboration), Chin. Phys. C 39, 093001 (2015).

[27] D. M. Asner et al., Int. J. Mod. Phys. A 24, 247 (2009).

[28] L. Köpke and N. Wermes, Phys. Rept. 174, 67 (1989).

[29] H. Kowalski and T. F. Walsh, Phys. Rev. D 14, 852 (1976).

[30] G. Ramalho and K. Tsushima, Phys. Rev. D 87, 093011 (2013).

[31] E. Kaxiras and E. J. Moniz, Phys. Rev. D 32, 695 (1985).

[32] F. Myhrer, Phys. Rev. C 74, 065202 (2006).

[33] H. B. Li, Front. Phys. 12, 121301 (2017); Erratum: Front. Phys. 14, 64001 (2019).

[34] M. Ablikim et al. (BESIII Collaboration), Phys. Rev. D 101, 012004 (2020).

[35] C. Carimalo, Int. J. Mod. Phys. A 2, 249 (1987).

[36] M. Claudson, S. L. Glashow and M. B. Wise, Phys. Rev. D 25,1345 (1982).

[37] M. Ablikim et al. (BESIII Collaboration), Phys. Rev. D 100, 051101(R) (2019).

[38] M. Gell-Mann, Phys. Rev. 125, 1067 (1962).

[39] O. W. Greenberg, Phys. Rev. Lett. 13, 598 (1964).

[40] M. G. Doncel, L. Michel and P. Minnaert, Nucl. Phys. B 38, 477 (1972).

[41] A. Z. Dubnickova, S. Dubnicka and M. P. Rekalo, Nuovo Cim. A 109, 241 (1996). 
[42] S. Dobbs et al., Phys. Lett. B 739, 90 (2014).

[43] S. Dobbs et al., Phys. Rev. D 96, 092004 (2017).

[44] M. Ablikim et al. (BESIII Collaboration), [arXiv:2103.12486 [hep-ex]].

[45] M. Ablikim et al. (BESIII Collaboration), Phys. Lett. B 817, 136328 (2021).

[46] A. Bianconi, E. Tomasi-Gustafsson, Phys. Rev. Lett. 114, 232301 (2015).

[47] I. T. Lorentz, H. W. Hammer, U. G. Meissner, Phys. Rev. D 92, 034018 (2015).

[48] F. Gross, G. Ramalho, K. Tsushima, Phys. Lett. B 690, 183 (2010).

[49] G. Ramalho, K. Tsushima, A. W. Thomas, J. Phys. G 40, 015102 (2013).

[50] G. Eichmann et al., Prog. Part. Nucl. Phys. 91, 1 (2016).

[51] J. P. Lees et al. (BaBar Collaboration), Phys. Rev. D 87, 092005 (2013).

[52] R. R. Akhmetshin et al. (CMD-3 Collaboration), Phys. Lett. B 759, 634 (2016).

[53] R. L. Jaffe and F. Wilczek, Phys. Rev. Lett. 91, 232003 (2003).

[54] G. Ramalho, M. T. Peña and K. Tsushima, Phys. Rev. D 101, 014014 (2020).

[55] D. Bisello et al. (DM2 Collaboration), Z. Phys. C 48, 23 (1990).

[56] B. Aubert et al. (BABAR Collaboration), Phys. Rev. D 76, 092006 (2007).

[57] M. Ablikim et al. (BESIII Collaboration), Phys. Rev. D 97, 032013 (2018).

[58] M. Ablikim et al. (BESIII Collaboration), Phys. Rev. Lett. 120, 132001 (2018).

[59] M. Ablikim et al. (BESIII Collaboration), Phys. Rev. D 103, 012005 (2021).

[60] M. Ablikim et al. (BESIII Collaboration), Phys. Lett. B 820, 136557 (2021).

[61] H. X. Chen, W. Chen, X. Liu and S. L. Zhu, Phys. Rep. 639, 1 (2016).

[62] M. Ablikim et al. (BESIII Collaboration), Phys. Rev. Lett. 124, 032002 (2020).

[63] L. Maiani, V. Riquer, F. Piccinini and A. D. Polosa, Phys. Rev. D 72, 031502 (2005).

[64] X. Liu, X. Q. Zeng and X. Q. Li, Phys. Rev. D 72, 054023 (2005).

[65] S. L. Zhu, Phys. Lett. B 625, 212 (2005).

[66] F. E. Close and P. R. Page, Phys. Lett. B 628, 215 (2005).

[67] M. Ablikim et al. (BESIII Collaboration), [arXiv:2108.02410 [hep-ex]]. 